\title{
Utilization of New Definitions to Calculate Overall Equipment Effectiveness (OEE) for Air Compressors: A Case Study
}

\author{
Mostafa Larky \\ Department of Mechanical Engineering \\ Iran University of Science and Technology \\ Tehran, Iran
}

\author{
Hamidreza Javidrad \\ Department of Mechanical Engineering \\ Iran University of Science and Technology \\ Tehran, Iran
}

\begin{abstract}
In recent Decade, utilization of management and engineering indicators has become a common task. Among these indicators, Mean Time Between Failure (MTBF), Mean Time To Repair (MTTR) and Overall Equipment Effectiveness (OEE) are more popular. Iran Khodro as the largest car manufacturer company in Iran and in the Middle East to optimize its production lines, electricity has been substituted by compressed air power. To achieve this target, this company launched compressed air department that has 50 air compressors including centrifugal and screw types and this number was reached gradually through the years. In this paper, to investigate the status of the equipment and report to department managers OEE index has been used. Since this indicator is more commonly used for systems and equipment that has a specific production schedule, this paper by proposing new definitions and changes in OEE parameters, calculation of this index for one compressor has become possible. By having this index for each compressor, one can analyze the performance of each compressor alone or in comparison with the others and as a case study the OEE of ten compressors belonged to the compressed air department since march to august of 2017 have been calculated and analysis has been reported.
\end{abstract}

Keywords: Overall Equipment Effectiveness (OEE), Air Compressor, Effective Maintenance, Equipment Status Report.

\section{INTRODUCTION}

Equipment analysis due to the variety in the operation of air compressors, such as centrifugal or screw type with different working pressure, and also the study of their operation since march to august of 2017 in terms of the number, timing and impact of downtime and efficiency of each Compressor Brigade for Iran Khodro Company will be performed in terms of the amount of compressed air produced in proportion to the energy consumption of a specific compressor using the calculation of the relevant indices.

Maintenance indexes have definite descriptions in all international references that will be used to compute them in this paper, but what matters is that for equipment with different definitions, the overall equipment efficiency (OEE) index can have different computational methods that can be found in This paper and have been computed for air compressors. Also, the technical information of the equipment that have been examined are discussed in detail.

In this paper, the analysis of the compressors used by Tehran Iran Khodro Company in terms of efficiency and calculation of maintenance indicators, as well as management indicators such as OEE index have been introduced, as well as compressors have been rejected in terms of quality.

\section{INTRODUCTION OF COMPRESSED AIR DEPARTMENT}

Iran Khodro Company as the largest car manufacturer in Iran and Middle East for the provision of compressed air for car production lines employs 50 air compressors in 7 and 10 bar pressure range which 10 compressors have been chosen as a case study. Since Iran Khodro is active in three working shifts and 24 hours a day, it is necessary that the working pressure of the production lines has been supplied constantly by the company's compressed air department at all working hours.
The compressors of this department based on the requirements of production lines are divided into two groups, compressors to generate 7 and 10 bar pressure. These compressors are from popular companies such as Samsung, Ingersoll rand, Atlas Copco, CompAir and Pars which produce compressed air in standard and oil free (high) quality. Standard quality air is used in body shops, assembly shops and rims shops where a little oil in the compressed air will not cause problem or fault. Oil free or high quality air is used in paint shop to power robots which are painting the body of the car.

\section{METHODOLOGY}

Effect of the efficiency of each compressor for the compressed air department of Iran Khodro in terms of the amount of compressed air and its energy consumption, as well as analysis based on the failure rates and their repairs and overhauls costs based on the calculation of relevant indicators such as PM rate, range rate, mean time to repair (MTTR), mean time between failure (MTBF), mean time to failure (MTTF), availability (AV), and finally the OEE which is a Management Indicator [1-3].

how to calculate the OEE index according to equation (1), rate of quality $(\mathrm{Q})$ and performance rate $(\mathrm{P})$ for the compressors of this company have been calculated.

$$
O E E=A v * P * Q
$$

Since the technical information, operating conditions and performance of the equipment under investigation are monitored, they are used for use in the formulas of the introduced indicators.

As shown in Equation 1, OEE index is calculated from the product of the three factors. If we want to calculate the total OEE for the compressed air department, then we can assume that the values of the parameters except $p$ have the value of 1 . 
The value of $p$ is reduced to 92 percent of its value due to the leakage measurement of the system, so for $p$, the value is 0.92 , and according to equation (1), OEE with a value of 0.92 can be calculated, which according to the system conditions is reasonable.

Since in this paper, the computation and expression of compressors effectiveness are considered, we assume the system boundary as compressor itself. In this method, the calculation of the factor $\mathrm{Q}$ will have a value of 1 . Because the point of measurement of the volumetric flow of air is just at the outlet of the compressor, which is located on the boundary and there is no rejected air at this point. On the other hand, various factors have to be considered in order to calculate the effectiveness of compressors.

It should be noted that in Iran Khodro's site, almost all of its production lines are operating 24 hours a day in three shifts. On the other hand, based on the market demand, it is necessary for production management to declare shifts in a number of production halls of the company at a number of scheduled holidays. Also, due to the fact that the distribution network of compressed air in Iran Khodro's site is continuous and there is no possibility of separating compressed air consumption of a hall, the process of producing and distributing compressed air is an uninterrupted process throughout the year. In the event of momentarily interruption of compressed air, some processes or equipment for production and support will be experiencing serious disturbance and damage. According to the above, in the calculations of the indices we have to consider the scheduled time as 365 days a year and 24 hour a day. In fig. (1) the suggested approach to define factors has been demonstrated.

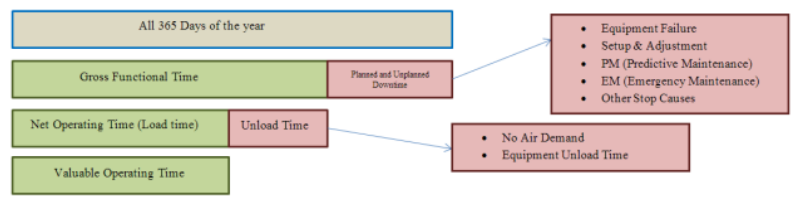

Figure 1. Suggested Approach.

In case of availability calculation equation (2) is suggested to use for air compressors.

$$
A v=\frac{(365 \text { Days of year }- \text { Downtimes })}{(356 \text { days of year })}
$$

As shown in equation (3), the parameter $\mathrm{P}$ is result of the production time of a net output of equipment to its entire availability time. In this paper, the net output of a compressor consists of the amount of compressed air produced by the compressor in a given time period in real working conditions. In other words, increasing the parameter $\mathrm{P}$ depends on the maximum production of compressed air of equipment during the entire time of its availability.

$$
P=\frac{(\text { Load Time })}{(365 \text { Days of year }- \text { Downtimes })}
$$

The important thing to note here is that all references that are related to total productive maintenance have focused on taking the planned net time into consideration. This is because the Av index increases dramatically by eliminating unplanned times for the production of an equipment (production planned time which is in the denominator of the Av index). While by the explanation given in this article is inevitably limited to consider the maximum possible time for working hours of equipment. It is natural that in this situation, any operation of the device despite its availability, due to internal or external factors, will all negatively affect the parameter P. Since one of the purpose of this paper is to calculate OEE as close as possible to its real value. To do so it has been recommended to multiply it by capacity coefficient (equation (4)).

$$
\text { Capacity Coefficient }=\frac{(\text { Actual Capacity })}{(\text { Nominal Capacity })}
$$

And by using equation (4), equation (5) can be achieved.

$$
P=(\text { Equation }(3)) *(\text { Equation }(4))
$$

Therefore by the explanations above, the suggested OEE equation to calculate for air compressors can be demonstrated as following equation (6) after simplification.

$$
O E E=\frac{(\text { Load Time }) *(\text { Capacity coefficient })}{(365 \text { Days of year })}
$$

Equation (6) has been resulted from putting equation (2), (3) and (5) and the value of quality which is one due to the mentioned reasons, into equation (1).

\section{RESULT AND DISCUSSION}

In this section all the data that are needed to investigate the status of equipment. The information of compressors that has been studied including manufacturing date, working pressure and quality of demanded compressed air are available in table (1). In this table demanded quality shows the criticality of compressed air quality produced by compressors in case of existence of oil in the compressed air. That actually is the reason of using oil free compressors to satisfy these demands. In the figure (2), a pie chart of compressors percentage by manufacturing date that is belonging to the compressed air department has been shown. In the fig.(2), it is demonstrated that most of the compressors have the age of at least 12 years. This figure includes all 50 compressors of the compressed air department.

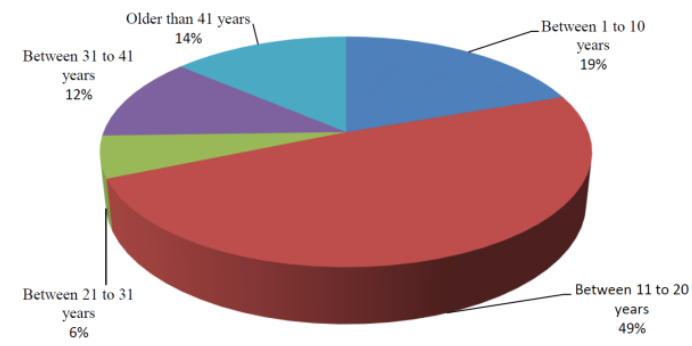

Figure 2. Pie chart of compressors percentage based on age. 
Table 1. Information of Compressors

\begin{tabular}{|c|c|c|c|}
\hline $\begin{array}{c}\text { Compressor } \\
\text { Number }\end{array}$ & $\begin{array}{c}\text { Manufacturing } \\
\text { date }\end{array}$ & $\begin{array}{c}\text { Demanded } \\
\text { quality }\end{array}$ & $\begin{array}{c}\text { Working } \\
\text { pressure }\end{array}$ \\
\hline 1 & 2015 & Standard & 7 bar \\
\hline 2 & 2015 & Standard & 7 bar \\
\hline 3 & 2002 & High & 7 bar \\
\hline 4 & 1996 & Standard & 7 bar \\
\hline 5 & 1998 & High & 10 bar \\
\hline 6 & 2000 & High & 7 bar \\
\hline 7 & 1971 & High & 7 bar \\
\hline 8 & 2009 & Standard & 7 bar \\
\hline 9 & 2015 & Standard & 7 bar \\
\hline 10 & 1983 & High & 7 bar \\
\hline
\end{tabular}

As it is shown in table (1), the age of the compressors is between 3 to 47 years. Type of the first three is centrifugal and the rest are screw. In the table (2), data for calculating OEE index including planned working time of compressors which is six months, total downtimes, load time have been shown.

Table 2. Required data

\begin{tabular}{|c|c|c|c|}
\hline $\begin{array}{c}\text { Compressor } \\
\text { Number }\end{array}$ & $\begin{array}{c}\text { Planned } \\
\text { time(hr) }\end{array}$ & $\begin{array}{c}\text { Total } \\
\text { downtime(hr) }\end{array}$ & $\begin{array}{c}\text { Load } \\
\text { time(hr) }\end{array}$ \\
\hline 1 & 4464 & 55 & 3588 \\
\hline 2 & 4464 & 55 & 3744 \\
\hline 3 & 4464 & 65 & 3059 \\
\hline 4 & 4464 & 2389 & 789 \\
\hline 5 & 4464 & 60.75 & 1496 \\
\hline 6 & 4464 & 1264.75 & 1686 \\
\hline 7 & 4464 & 55 & 2015 \\
\hline 8 & 4464 & 45.5 & 2002 \\
\hline 9 & 4464 & 55 & 1821 \\
\hline 10 & 4464 & 55 & 2542 \\
\hline
\end{tabular}

Since the calculation of $\mathrm{P}$ factor needs computing capacity coefficient and to do so must extract nominal and actual capacity from table (3).

Table 3. Nominal and actual capacity

\begin{tabular}{|c|c|c|}
\hline $\begin{array}{c}\text { Compressor } \\
\text { Number }\end{array}$ & $\begin{array}{c}\text { Nominal } \\
\text { capacity(m^3/min) }\end{array}$ & $\begin{array}{c}\text { Actual } \\
\text { capacity(m^3/min) }\end{array}$ \\
\hline 1 & 83 & 79 \\
\hline 2 & 83 & 79 \\
\hline 3 & 55 & 51 \\
\hline 4 & 43 & 35 \\
\hline 5 & 30 & 26 \\
\hline 6 & 32 & 28 \\
\hline 7 & 32 & 28 \\
\hline 8 & 44 & 38 \\
\hline 9 & 70 & 68 \\
\hline 10 & 32 & 28 \\
\hline
\end{tabular}

Finally by using equations (2) to (6) and tables (1) to (3), OEE index and its factor can be achieved for mentioned equipment as following table (table (4))

Table 4. Final results

\begin{tabular}{|c|c|c|c|}
\hline $\begin{array}{c}\text { Compressor } \\
\text { Number }\end{array}$ & Av & P & OEE \\
\hline 1 & 0.99 & 0.77 & 0.76 \\
\hline 2 & 0.99 & 0.81 & 0.8 \\
\hline 3 & 0.99 & 0.64 & 0.64 \\
\hline 4 & 0.46 & 0.31 & 0.14 \\
\hline 5 & 0.99 & 0.29 & 0.29 \\
\hline 6 & 0.72 & 0.46 & 0.33 \\
\hline 7 & 0.99 & 0.4 & 0.39 \\
\hline
\end{tabular}

\begin{tabular}{|c|c|c|c|}
\hline 8 & 0.99 & 0.39 & 0.39 \\
\hline 9 & 0.99 & 0.4 & 0.4 \\
\hline 10 & 0.99 & 0.5 & 0.5 \\
\hline
\end{tabular}

As it is shown in table (4), some of the OEE indexes are very low which to analyze and explain the reasons must compute other indicators such as MTTF and MTBF. Results have been shown in table (5). These indicators have been calculated by using equation (7) and (8).

$$
M T T F=\frac{(\text { Planned time })-(\text { Repair time })}{\text { Number of failures }}
$$

$$
M T B F=\frac{(\text { Planned time })}{\text { Number of failures }}
$$

Table 5. Analysis required data

\begin{tabular}{|c|c|c|c|}
\hline $\begin{array}{c}\text { Compress } \\
\text { or } \\
\text { Number }\end{array}$ & MTTF & MTBF & $\begin{array}{c}\text { Number } \\
\text { of failures }\end{array}$ \\
\hline 1 & $\infty$ & $\infty$ & 0 \\
\hline 2 & $\infty$ & $\infty$ & 0 \\
\hline 3 & 2229.5 & 2232 & 2 \\
\hline 4 & 1060 & 2232 & 2 \\
\hline 5 & 2230.63 & 2232 & 2 \\
\hline 6 & 1085.75 & 1488 & 3 \\
\hline 7 & $\infty$ & $\infty$ & 0 \\
\hline 8 & 4463.5 & 4464 & 1 \\
\hline 9 & $\infty$ & $\infty$ & 0 \\
\hline 10 & $\infty$ & $\infty$ & 0 \\
\hline
\end{tabular}

During the investigations and data collection, the number of failures and repair time of the compressors from March to august of 2017 have been showed in table 5 .

In this paper, compressors that have been studied have different OEE index that can be analyzed more accurately according to the MTTF and MTBF index. MTTF and MTBF indices are infinite when there is no failure during the time interval. When at least one breakdown leads to a stop, the most ideal condition is to fix the failure at the shortest possible time, in which case the MTTF will be close to the planned time, which can be equal to the MTBF index.

For example, Compressor number 1 and 2 have a planned working time of 4464 hours between march to agaust of 2017, but their run times were about 3,600 hours, considering that during this period they did not stop because of breakdowns or PMs. Therefore, they have a high AV index, and during this time compressors have been almost always on and on load. it must be taken into account that these equipment as shown in table 1 are not old, so their capacity coefficient has a high value. As a result, the OEE index is desirable. For compressor number 1 , the only reason for the OEE index deviation can be related to the time that no air was demanded.

The other examples are compressors number 7, 9 and 10 which their operating times do not have stops because of CMs and EMs but the time to use the equipment was much less than the planned time Which means the most ideal MTTF and MTBF index and a lower value in OEE index. as it has been said earlier the low OEE is directly related to the low usage of the equipment. 
Another example in compressor number 6 as it can be seen in the table 2, the load time of the equipment is 1686 which is less than the planned time. it must be taken into account that during this time it has done approximately 2000 hours of run. The equipment has failed and its operation has stopped 3 times in the assumed time interval. Downtime of the equipment includes more than 1200 hours of repair time which has a very effective influence in MTBF and MTTF indices. it can be concluded that this equipment has low effectiveness and low performance.

By the discussion above, it has been proven that proposed approach can be used to calculate and compare compressor conditions which lead to a great index to help managers make better decisions.

\section{CONCLUSION}

Unlike most maintenance and repair engineering indicators that cannot be investigated alone, the OEE index is an analytical and management evaluation indicator that, as stated, the product of the multiplier is the factor of accessibility, the rate of quality and the rate of performance. To make since the performance of the device is considered in this analysis, we consider the system boundary as the device itself. In this calculation, there is no $Q$ factor because the point of measurement of the volumetric flow is exactly at the outlet of the compressor and there is no rejection air. Therefore, in the calculations for the OEE index instead of having three components, only have two which are AV and P. Reviews have also been made to find the best performance rates. The results in separate tables describe the performance of all compressors according to the type of application and their working conditions, which will allow the respective managers to take care of the equipment and personnel involved in the maintenance planning and Have a clearer vision on this matter. As mentioned in methodology section, OEE of compressed air department is 0.92 which is good but about the OEE of the equipment, different values have been resulted. These low values can be explained by using other indicators such as MTTF and MTBF. For example compressor 1 has a run time of 3588 hours but its planned working time is 4464 hours in 24 hours and 3 shifts. Since it had no failure in the assumed time interval, the cause of this difference is mostly no air demands which by looking at the MTBF and MTTF , it is obvious that this equipment has no failure .It does need to be considered that these calculations are done for the first time at compressed air department in Iran Khodro Company.

\section{REFERENCES}

[1] Dal B., Tugwell P., Greatbanks R. (2000), Overall equipment effectiveness as a measure of operational improvement - A practical analysis, International Journal of Operations \& Production Management, Vol. 20 Issue: $12,1488-1502$.

[2] Muchiri P., Pintelon L. (2008), Performance measurement using overall equipment effectiveness (OEE): literature review and practical application discussion, 3517-3535.

[3] Relkar A.S., Nandurkar K.N. (2012), Optimizing \& Analysing Overall Equipment Effectiveness (OEE) Through Design of Experiments (DOE), Procedia Engineering Volume 38, 2973-2980. 